\title{
Composición corporal y rendimiento físico de jugadores de fútbol soccer universitario por posición de juego \\ Body composition and physical performance of college soccer by player's position \\ *Oswaldo Ceballos-Gurrola, **Fernando Bernal-Reyes, ${ }^{* * *}$ Marquelia Jardón-Rosas, *María Cristina Enríquez-Reyna, ***Jesús Durazo-Quiroz, ***María Gretel Ramírez-Siqueiros \\ *Universidad Autónoma de Nuevo León (México), ** Universidad de Sonora (México), ***Universidad Estatal de Sonora (México)
}

Resumen. Se propuso determinar la relación que existe entre la composición corporal y el rendimiento físico de jugadores de fútbol soccer universitario. Participaron 26 jugadores de sexo masculino con edad promedio de 20.7 años $(D T=1.7)$, pertenecientes a los equipos representativos de fútbol soccer de dos universidades mexicanas. Se midieron características morfológicas, de composición corporal y físicas para las posiciones de defensa, mediocampo y delantero. Se determinó el nivel de asociación entre las variables. Los resultados muestran que los defensas presentan mayor masa corporal, diámetro de húmero y circunferencia de cadera que los delanteros $(p<.05)$, no así respecto a los jugadores de mediocampo. No se presenta diferencia en la expresión de las capacidades físicas y del porcentaje de masa grasa de los jugadores por posición de juego $(p>.05)$. La masa grasa se asoció de manera directa con la velocidad $\left(.427^{*}, p<.05\right)$. Se encontraron diferencias en la masa ósea y corporal por posición de juego entre defensas, mediocampistas y delanteros. Los hallazgos sugieren la influencia de la composición corporal en el rendimiento físico de los participantes.

Palabras clave. Masa grasa, pliegues cutáneos, pruebas físicas, jugadores universitarios, fútbol.

Abstract. The aim of the study was to determine the relationship between body composition and physical performance in college soccer players. Twenty-six male players with an average age of 20.7 years $(S D=1.7)$, belonging to the representative soccer teams of two Mexican universities participated in the research. Morphological, body composition, and physical characteristics were measured for the defense, midfield, and forward positions. The level of association between variables was determined. The results show that defenders have greater body mass, humerus diameter, and hip circumference than forwards $(p<.05)$, whereas no differences were found in comparison to midfielders. There is no difference in players' expression of physical abilities and percentage of fat mass by playing position $(p>.05)$. Fat mass was directly associated with speed $\left(.427^{*}, p<.05\right)$. Differences were found in bone and body mass by playing position between defenders, midfielders, and forwards. The findings hint at an influence of body composition on participants' physical performance.

Key words. Fat mass, skinfolds, physical tests, college players, soccer.

\section{Introducción}

El fútbol soccer es el deporte con mayor difusión y también el más popular a nivel mundial, se caracteriza por ser una disciplina deportiva que demanda un gran esfuerzo físico, ya que combina resistencia con diversas fases explosivas. Se considera un deporte intermitente pues en ciertos momentos se realizan sprints a máxima velocidad, cambios de dirección, saltos, trote, correr hacia atrás, desacelerar e incluso permanecer simplemente en la misma posición (Burke, 2010), con un tiempo de duración de un partido que va desde los 90 hasta los 120 minutos. Para lograr el desarrollo de las capacidades físicas, se requiere un consumo energético elevado pues los entrenamientos reflejan un arduo trabajo en campo durante la fase de preparación (Nikolaidis, 2015). En promedio un partido de fútbol demanda al atleta un $75 \%$ de intensidad en su capacidad aeróbica, aunque también requiere del uso del sistema energético anaeróbico aláctico (Carling \& Orhant, 2010). La práctica del fútbol en la etapa universitaria es muy elevada entre la población estudiantil, sin embargo, solo un pequeño grupo a nivel nacional se dedica a este deporte de forma profesional, ya que las demandas académicas y el ritmo de vida le limitan en tiempo y dedicación. Por tanto, se practica más de forma recreativa y mayormente en fin de semana y durante las vacaciones (Gómez, Ruiz \& García, 2010). Los deportistas que se enfocan en una práctica consciente y disciplinada desarrollan

Fecha recepción: 31-10-19. Fecha de aceptación: 05-05-20 María Gretel Ramírez-Siqueiros

grethel.ramirez@ues.mx características morfológicas muy particulares, donde a simple vista se puede observar un mayor desarrollo muscular del tren inferior y un muy bajo porcentaje de grasa en contraste con el tren superior. Las capacidades físicas de fuerza, velocidad y potencia favorecen el desarrollo de la masa corporal magra, misma que es importante para ubicar mejor las posiciones del juego (Burke, 2010).

Durante el proceso de entrenamiento el deportista recibe dos tipos de estímulos denominados carga de entrenamiento (externa e interna), la externa es la parte tangible es decir la demanda física, mientras que la interna se refiere a la parte intangible como la respuesta fisiológica que obtiene el deportista ante el estímulo generado, el cual repercute morfofisiológica, biomecánica y psicosocialmente en los jugadores (Casamichana, San Román-Quintana, CallejaGonzález \& Castellano, 2013; Poveda \& Casamichana, 2017; Del Río-Valdivia, et al., 2017). Se ha observado que la misma carga externa repercutirá de diferente manera sobre la carga interna en dos individuos diferentes ya que cada uno desarrolla adaptaciones particulares en respuesta al entrenamiento (Manzi, Bovenzi, Impellizzeri, Carminati \& Castagna, 2013; Rodríguez, Montenegro \& Petro, 2019). La composición corporal de un atleta constituye una de las variables que puede influir notablemente en los resultados de su disciplina deportiva, sin embargo, es solo un aspecto más que se debe de tener en cuenta, ya que pudiera favorecer o limitar el rendimiento en determinado momento de su preparación física.

Es importante que los jugadores de fútbol soccer tengan un determinado porcentaje de masa grasa $(\% \mathrm{MG})$ para desempeñarse eficientemente y lograr su máximo potencial en el juego (Fernández, Kazarez,Agazzi \& Albín, 2014; Gardasevic, 
Bjelica \& Vasiljevic, 2019). El objetivo del presente estudio fue determinar la relación que existe entre la composición corporal y el rendimiento físico de jugadores de fútbol soccer universitario para medir si ésta se encuentra diferenciada en función de la posición de juego.

\section{Material y métodos}

Se presenta un estudio de corte transversal de tipo descriptivo-correlacional, no experimental. Se evaluaron $26 \mathrm{ju}-$ gadores de sexo masculino con una edad promedio de $20.7 \pm$ 1.7 años, pertenecientes a los equipos representativos de fútbol soccer de la Universidad Estatal de Sonora (UES) y Universidad de Sonora en México (UNISON).

\section{Procedimientos}

Los participantes aceptaron previamente su participación en el estudio y firmaron un consentimiento informado. La recogida de datos se efectuó en distintos días, pero en el mismo campo de su universidad, a la misma hora y en el mismo período de entrenamiento, en etapa previa a competencia. En cada sesión de levantamiento de datos se llevó un control de los sujetos evaluados, donde primeramente se realizó antropometría básica, la toma de los pliegues cutáneos y posteriormente se aplicaron las pruebas físicas. Se ha cumplido los principios éticos en relación con las personas que participan en el estudio, al contar con su consentimiento informado.

\section{Valoración antropométrica}

Para realizar la valoración antropométrica se aplicó el protocolo internacional establecido por Stewart, MarfellJones, Olds y de Ridder (2011). En dicho protocolo se tomaron mediciones básicas de peso, talla, talla sentado y envergadura, así como diámetros, perímetros y ocho pliegues cutáneos, considerando la estandarización de técnicas y procedimientos de la I.S.A.K. Sociedad Internacional para el desarrollo de la Cineantropometría (International Society for the Advancement of the Kinanthropometry (ISAK, 2001; Marfell-Jones, Olds, Stewart \& Carter, 2006). Para las mediciones se utilizó un estadiómetro marca SECA modelo 21, una báscula marca SECA modelo 634, una cinta antropométrica marca Rosscraft, un paquímetro marca Rosscraft y un plicómetro marca Harpender para la medición de los pliegues cutáneos. Las valoraciones en el presente estudio fueron realizadas por dos antropometristas nivel 2 certificados por ISAK. Se estimó el \%MG mediante la ecuación de Wilmore y Behnke (1969), ecuación que ha mostrado valores cercanos a los obtenidos por el método de referencia DEXA para jugadores futbolistas de élite (García, Zapata, Sáez, Yáñez \& Peñailillo, 2015).

Se utiliza la fórmula: $\mathrm{DC}=1,08543-0,000886 \times(\mathrm{AB})-$ $0,00040 \times(\mathrm{PM})$. Donde DC $=$ Densidad Corporal; $\mathrm{AB}=$ Pliegue Abdominal (mm); PM=Pliegue Pantorrilla Medial (mm); El \%MG se calculó a partir de valores de DC mediante la fórmula de Brozek (1963), de acuerdo con la siguiente ecuación: \%MG: [(4,57/D)-4,142] x100.

\section{Valoración física}

Para realizar la valoración física, los participantes reci- bieron la explicación de cada una de las pruebas, así como también se desarrolló un calentamiento de 15 minutos de duración, consistente en desplazamientos variados, movilidad articular, salidas y acciones explosivas propias del fútbol, tal y como lo tienen establecido los equipos de forma habitual. Las pruebas físicas se llevaron a cabo en un campo de fútbol de césped artificial, excepto la prueba de salto vertical donde la superficie debe de ser plana para no perjudicar al atleta a la hora de realizar el salto con la mayor libertad posible.

Prueba de agilidad de Illinois. La agilidad se evaluó mediante la aplicación de la prueba de Illinois (Vescovi \& McGuigan, 2008). La longitud del recorrido es de 10 metros y el ancho (distancia entre los puntos de inicio y final) es de 5 metros. Cuatro conos se usan para marcar el inicio, el final y los dos puntos de inflexión (A, B, C Y D como se muestran en la Figura 1). Otros cuatro conos se colocan en el centro a igual distancia. Una vez ubicados como indica el gráfico, se comienza la prueba en posición acostado boca abajo con las manos en el nivel del hombro, debiendo levantarse tan rápidamente como sea posible y recorrer la trayectoria del sistema (puede ser en cualquiera de los dos sentidos empezando en A o en D). No se debe golpear ningún cono al pasar por ellos. La prueba es completada y se detiene el cronómetro, cuando no hay conos golpeados y se cruza la línea de final. La interpretación de la prueba se realiza de la siguiente manera: Excelente ( $<15.2 \mathrm{~s})$, sobre promedio (15.2-16.1 s), promedio (16.2-18.1 s), bajo promedio (18.2-18.8 s), pobre ( $>18.8 \mathrm{~s})$.

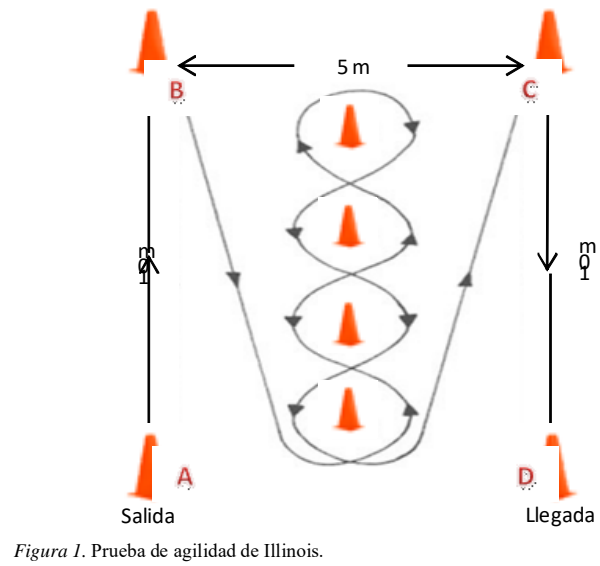

Prueba de velocidad T-test. La velocidad se evaluó mediante la prueba T-test (Badenhorst, 1998), la cual permite medir qué tan rápido se puede cambiar de dirección y con precisión. Para iniciar la prueba, se deben ubicar los conos como se señalan en la Figura 2, iniciando en el cono A, correr rápidamente hasta el cono $\mathrm{B}$ y tocarlo con la mano derecha, posteriormente deberá dirigirse al cono $\mathrm{C}$, corriendo de costado y tocarlo con la mano izquierda. Luego dirigirse al cono $\mathrm{D}$ (corriendo de costado) para tocarlo con la mano derecha, vuelve al cono $\mathrm{B}$ para tocarlo con la mano izquierda y vuelve corriendo de espaldas al cono A. Se efectúa la prueba tres veces y se anota el mejor de los tres tiempos, para la interpretación se toma como referencia los siguientes criterios: Excelente $(<9.5 \mathrm{~s})$, buena $(9.5-10.5 \mathrm{~s})$, promedio $(10.5-11.5 \mathrm{~s})$, pobre $(>11.5 \mathrm{~s})$.

Prueba de Course Navette. Se utiliza esta prueba para 


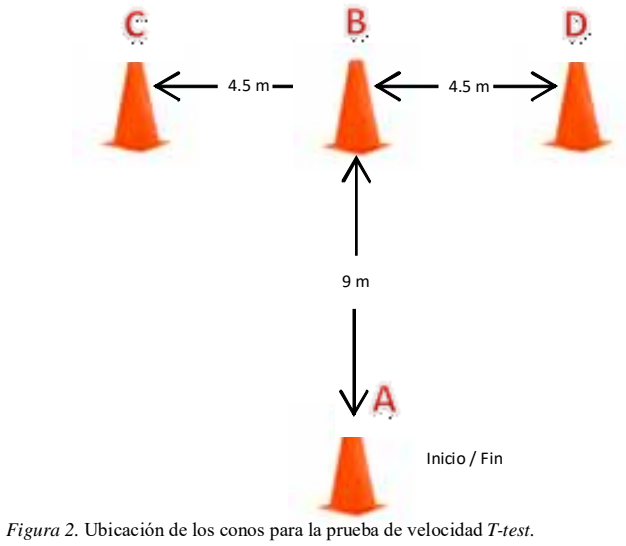

evaluar la resistencia cardiorrespiratoria (Leger \& Lamber, 1982). Considerando que dicha prueba es aplicable a sujetos con entrenamiento medio o bajo, se registra la velocidad alcanzada en esta prueba calculando el $\mathrm{VO}_{2}$ máx de la siguiente manera: $\mathrm{VO}_{2}$ máx $=(31.025)+(3.238 * \mathrm{X})-(3.248 * \mathrm{~A})+$ $\left(0.1536^{*} \mathrm{~A} * \mathrm{X}\right)$. Donde $\mathrm{X}=$ Velocidad a la que se detuvo el sujeto; $\mathrm{A}=\mathrm{Edad}$. A este respecto, se considera que, para sujetos mayores de 18 años, siempre se aplica el valor de 18 en ese rubro. Un resultado de $\mathrm{VO}_{2}$ máx $<25 \mathrm{ml} / \mathrm{kg} / \mathrm{min}$ es considerado bajo, de 25 a $33 \mathrm{ml} / \mathrm{kg} / \mathrm{min}$ regular, 34 a $42 \mathrm{ml} / \mathrm{kg} / \mathrm{min}$ como media, de 43 a $52 \mathrm{ml} / \mathrm{kg} / \mathrm{min}$, buena; y excelente si es $>52 \mathrm{ml} / \mathrm{kg} / \mathrm{min}$.

Prueba de salto vertical. Esta prueba consiste simplemente en saltar tan alto como se pueda (Sargent, 1921). La prueba comienza con el participante parado directamente debajo del dispositivo de registro. Se toma una medición inicial en la que el participante se extienda tan alto como sea posible con sus pies planos sobre el suelo, el dispositivo se ajusta de modo que el participante pueda alcanzar la varilla horizontal más baja, realiza una flexión de piernas, balancea ambos brazos hacia abajo y atrás, los gira rápidamente hacia adelante y salta tan alto como sea posible. En el punto más alto debe tocar la varilla en el dispositivo de salto vertical. La altura del salto vertical se define como la distancia entre la marca inicial tomada de pie y la cima del salto. Se realizan tres ensayos y se registra el salto más alto. Entre cada ensayo se permite un período de recuperación breve. La interpretación de parámetros para hombres en el salto vertical se realiza de la siguiente manera: Excelente $(>70 \mathrm{~cm})$, Encima de la media $(56-70 \mathrm{~cm})$, Promedio $(41-55 \mathrm{~cm})$, Por debajo del promedio $(31-40 \mathrm{~cm})$, Pobre $(<30 \mathrm{~cm})$.

\section{Análisis estadístico}

Para determinar la normalidad de los datos se aplicó la prueba de Shapiro-Wilk con corrección de Levene, considerando que no se obtuvo una distribución normal para todas las variables. Los análisis comparativos entre grupos por posición de juego (defensa, mediocampo y delantero) se realizaron mediante la prueba de Kruskal-Wallis con corrección de Bonferroni, con un valor de significancia de .05. Para comparar la relación entre las variables morfológicas, composición corporal y de rendimiento físico, se utilizó el coeficiente de correlación de Spearman. El análisis estadístico de los datos se realizó mediante el programa SPSS (V24.0; SPSS, Ins; Chicago, IL, USA).

\section{Resultados}

En la Tabla 1 se muestran las características morfológicas básicas de los jugadores por posición de juego. Al realizar la comparación entre los grupos se obtuvo diferencia en peso corporal $\left(\mathrm{Chi}^{2}{ }_{2}=6.161, p=.04\right)$, el diámetro humeral $\left(\mathrm{Chi}_{2}{ }_{2}=\right.$ $8.003, p=.018)$ y en el pliegue subescapular $\left(C h i^{2}{ }_{2}=6.456, p\right.$ $=.04)$; con tendencia a la significancia al respecto del perímetro de cadera $\left(\mathrm{Chi}_{2}{ }_{2}=5.408, p=.067\right)$. También se indican los valores promedio para los ocho pliegues cutáneos y la sumatoria de éstos, así como el \% MG por posiciones de juego.

Tabla 1

Características morfológicas y de masa grasa de los jugadores por posición de juego

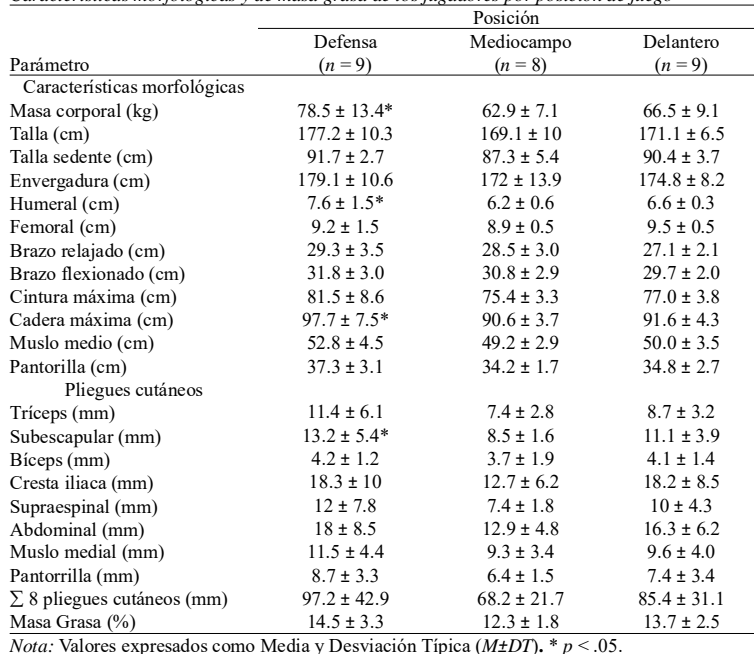

Se obtuvo que el pliegue cutáneo subescapular de los defensas es mayor $(p<.05)$ respecto al de los jugadores de mediocampo, no así respecto a los delanteros el cual es similar. No se obtuvo diferencia en la sumatoria de los ocho pliegues al determinarlo por posición de juego. Al analizar las variables físicas por posición de juego no se identificaron diferencias entre las capacidades físicas evaluadas, por posiciones de juego. Los datos se muestran en la Tabla 2.

\begin{tabular}{|c|c|c|c|}
\hline Parámetro/Posición & $\begin{array}{l}\text { Defensa } \\
(n=9)\end{array}$ & $\begin{array}{c}\text { Mediocampista } \\
(n=8)\end{array}$ & $\begin{array}{c}\text { Delantero } \\
(n=9)\end{array}$ \\
\hline Agilidad (s) & $15.3 \pm 0.5$ & $14.9 \pm 0.6$ & $15.1 \pm 0.8$ \\
\hline Velocidad (s) & $11.2 \pm 0.7$ & $10.5 \pm 0.8$ & $10.4 \pm 0.7$ \\
\hline Resistencia ( $\mathrm{VO}_{2}$ máx, $\mathrm{ml} / \mathrm{kg} / \mathrm{min}$ ) & $41.3 \pm 4.6$ & $41.9 \pm 4.0$ & $44.5 \pm 4.2$ \\
\hline Salto vertical $(\mathrm{cm})$ & $61.4 \pm 7.6$ & $59.5 \pm 10.5$ & $67.5 \pm 6.7$ \\
\hline
\end{tabular}

Los resultados de las diversas pruebas físicas aplicadas a los jugadores muestran que en promedio los jugadores presentan agilidad excelente $(<15.2 \mathrm{~s})$, velocidad dentro del rango promedio (10.5-11.5 s), resistencia aeróbica buena (43 a $52 \mathrm{~L} / \mathrm{min})$ y salto vertical $(56-70 \mathrm{~cm})$ por encima de la media. En la Tabla 3 se muestran los resultados de correlacionar el $\% \mathrm{MG}$, la sumatoria de 8 pliegues y el rendimiento deportivo (agilidad, velocidad, resistencia y salto vertical) en la muestra completa. Las pruebas físicas no mostraron correlación con la sumatoria de pliegues cutáneos y \% $\mathrm{MG}(p>.05)$.

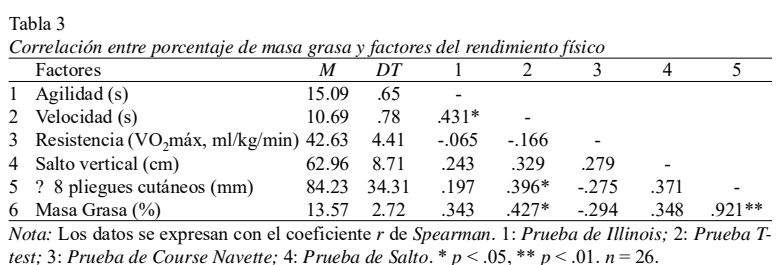


Al realizar análisis posteriores en relación al grado de asociación entre las variables por posición de juego se encontraron diferentes hallazgos. En la posición de defensa se encontró asociación inversa entre el porcentaje de grasa corporal y la resistencia aeróbica $(r=-.848, p<.01)$. Este dato no se presenta en tablas.

\section{Discusión}

El propósito de este estudio fue determinar la relación entre la estructura morfológica, la composición corporal de los jugadores de fútbol universitario y sus capacidades físicas. La correlación entre la composición corporal y el rendimiento deportivo se ha investigado por años, ya que se pretende dar a conocer si la estructura morfo física de un atleta influye en el desempeño de su disciplina deportiva (Peña-Reyes, 2017; Ryan-Stewart, Faulkner \& Jobson, 2018). Aunque ciertamente se observaron asociaciones entre indicadores de la composición corporal y la capacidad física, en esta muestra, la posición de juego generó diferencias al respecto de aspectos morfológicos pero no de las capacidades físicas evaluadas. Esto coincide con lo reportado por García-Pinillos, Ruiz-Ariza y Latorre-Román (2015), quienes concluyen que la condición física no presenta rasgos marcados, específicos de una determinada posición de juego cuando se trata de jugadores jóvenes y nivel no profesional, ya que lo que se persigue a esas edades es una formación integral y desarrollo lo más amplio posible, tanto a nivel físico como técnico-táctico.

A pesar de la existencia de varios trabajos que han estudiado la composición corporal en las modalidades de fútbol y baloncesto, se muestra poca evidencia en el ámbito universitario según lo expresan Gil-Gómezy Verdoy (2011), quienes evaluaron a jugadores españoles universitarios que presentaron un $14.4 \% \mathrm{MG}$, el cual es un porcentaje muy similar al obtenido en la presente investigación. Así mismo el estudio realizado por Hetzler, Schroeder, Wages, Stickley y Kimura (2010), quienes obtuvieron un $13.9 \%$ MG en jugadores universitarios y realizaron la comparación con jugadores de alto rendimiento quienes mostraron un porcentaje de $10.7 \%$. Sin embargo, otros autores establecen un rango más conservador de entre 8 y $10 \%$ en jugadores profesionales (Casajús, 2001; Tomkinson, Popoviæ\& Martin, 2003). Ante este referente, el \%MG de los jugadores que participaron en el presente estudio es elevado, pero se asemeja al reportado para jugadores universitarios, lo cual puede explicarse debido al nivel amateur de la presente muestra.

Por otro lado, al analizar la composición corporal de los jugadores por posición de juego, no se obtuvo diferencias. Estudios similares que han evaluado características morfológicas de jugadores de fútbol, muestran una estructura corporal promedio con predominancia del componente magro, pero al ser analizadas por posición de juego se expresan diferencias en las posiciones defensivas respecto a las ofensivas, lo cual puede observarse cuando se trata de futbolistas de alto nivel, siendo esta información de relevancia para fines de la optimización morfológica (HenríquezOlguín, Báez, Ramírez-Campillo \& Cañas, 2018). En el estudio realizado por Zúñiga, Osorio, Toledo y Herrera (2018), evaluaron el somatotipo de futbolistas mexicanos profesionales de diferente nivel competitivo y concluyeron que, los cambios en la estructura corporal se aprecian a medida que los jugadores de futbol avanzan en edad y en el nivel de juego. Así también Caballero, Carrasco, De León, Candia y Ortíz, (2019) argumentaron que sus resultados similares, podrían indicar que las diferencias en la distribución del somatotipo son observadas en futbolistas de alto rendimiento. Tomando en cuenta estos hallazgos debemos considerar que los jugadores del presente estudio son jugadores de nivel universitario, no de nivel profesional aún y que posiblemente el nivel de entrenamiento no ha condicionado la diferenciación en su posición en la cancha.

En el presente estudio se obtuvieron diferencias en algunas variables antropométricas al analizarlas por posiciones. El peso corporal de los defensas es mayor que el de los jugadores de mediocampo $(p<.05)$, así como el pliegue subescapular, sus diámetros de húmero y cadera. Esto atiende a las características necesarias de su morfología en su posición en el campo de impedir que los adversarios se acerquen a portería y marquen gol. Parece ser que entre más defensiva es la posición de juego, menos intensa es la actividad física que se realiza, lo cual se ha relacionado con una mayor cantidad de masa grasa corporal, ya que el gasto energético en este grupo de jugadores es menor (Jorquera, et al., 2013).

El pliegue subescapular y el \%MG como indicadores de la composición corporal fueron evaluados por Gardasevic et al. (2019), con jugadores de élite pertenecientes a tres clubs de fútbol de Montenegro con edad promedio de 22.73 años $(D T=4.66)$. En esa muestra reportaron diferencias en el pliegue subescapular y el \%MG, aunque no se correlacionaron otras variables de tipo físico, los autores declararon que valores bajos en la composición corporal eran deseables para un desempeño óptimo en los jugadores.

Es importante considerar la función dentro del campo de juego a la hora de interpretar la composición corporal y describir el nivel de rendimiento, en este sentido, la asociación significativa y positiva obtenida en la presente investigación entre el \%MG y la velocidad es una relación que ha sido reportada por Clael et al. (2018), quienes evaluaron a jugadores de fútbol brasileños universitarios (20.9 años, $D T=1.84$ ). En ese estudio los autores señalaron que la composición corporal es un factor determinante en las pruebas físicas de los atletas, siendo la adiposidad un factor que influye negativamente en la aptitud física.

En esta muestra, como parte de la evaluación de sus capacidades físicas, los jugadores muestran una velocidad dentro del promedio, sin embargo, un bajo nivel de $\mathrm{VO}_{2}$ máx promedio al ser comparado con el reportados en otros estudios, con valores de entre 55.94 y $58.38 \mathrm{ml} / \mathrm{kg} / \mathrm{min}$ (Sánchez, Ureña, Salas, Blanco \& Araya, 2011; Vidarte, Castiblanco, Villa \& Ortega Parra, 2019). Esto refleja el reducido nivel de entrenamiento, falta de madurez y eficiencia del sistema cardiorespiratorio. Se consideran aspectos a tomar en cuenta en función de la edad y nivel competitivo actual de los jugadores evaluados. Eso debe contemplarse cuando se pretenda proyectar a los equipos a un nivel competitivo. En futuras investigaciones se sugiere evaluar a un mayor número de jugadores por posición de juego para poder determinar con propiedad la relación del componente 
morfofisiológico y el rendimiento acorde a su función del campo de juego. Se considera que no fue posible establecer en el presente estudio, específicamente para la posición de portero, dado el reducido número de sujetos en dicha posición.

\section{Conclusiones}

La composición corporal muestra una influencia en el desempeño deportivo de los jugadores de fútbol universitario que participaron en el estudio. El elevado \%MG corporal es un condicionante de la velocidad, aspecto de vital importancia en la disciplina del fútbol, considerando que este grupo se encuentran aún en proceso de maduración morfofisiológica así como también en la etapa previa para obtener su posible participación en el nivel profesional.

\section{Referencias}

Badenhors, E. (1998). 'N Keuringsmodel vir talentidentifisering by 16 -jarige sokkerspelers. [A selection model for talent identification for 16-year-old soccer players. South Africa.] Master's thesis, North West Univ. 52p.

Brozek, J., Grande, F., Anderson, J. T., \& Keys, A. (1963). Densitometric analysis of body composition: revision of some quantitative assumptions. Annals of the New York Academy of Sciences, 110(1), 113-140. https:// doi.org/ 10.1111/j.1749-6632.1963.tb17079.x

Burke, L. (2010). Nutricion en el Deporte/Nutrition in Sport: Un Enfoque Práctico/a Practical Approach. (6 $6^{\mathrm{a}}$ ed). Ed. Médica Panamericana. Australia.

Caballero-Ruíz A., Carrasco-Legleu C., De León L., CandiaLuján R., \& Ortiz-Rodríguez B. (2019). Somatotipo de mujeres futbolistas universitarias por posición en el terreno de juego. Retos. Nuevas tendencias en Educación Física, Deporte y Recreación, (36), 228-230.

Carling, C. \& Orhant E. (2010). Variation in body composition in professional soccer players: interseasonal and intraseasonal changes and the effects of exposure time and player position. The Journal of Strength \& Conditioning Research, 24(5), 1332-1339. https:// doi.org/10.1519/JSC.0b013e3181cc6154.

Casajús, J.A. (2001). Seasonal variation in fitness variables in professional soccer players. Journal of Sports Medicine and Physical Fitness, 41(4), 463-469.

Casamichana, D., San Román-Quintana J., Calleja-González J., \& Castellano, J. (2013). Utilización de la limitación de contactos en el entrenamiento en fútbol: ¿afecta a las demandas físicas y fisiológicas? RICYDE. Revista Internacional de Ciencias del Deporte, 9(33): 208-221. https:/ / doi.org/10.5232/ricyde2013.03301.

Clael, S., de Oliveira Castro, H., Júnior, W. S. P., Neves, R. V. P., Rosa, T. S., da Silva Aguiar, S., ... Bezerra, L. (2019). Negative association between quantities of body fat and physical fitness of university football players. Sport Sciences for Health, 15(1), 191-195. https:// doi.org/ 10.1007/s11332-018-0509-3.

Del Rio-Valdivia, J. E., Salazar, C. M., Cuevas-Romo, J., Andrade-Sánchez, A. I., Flores-Moreno, P. J.,.. Ramos
Carranza, I. G. (2017). Diferencias en el OBLA en jugadoras de fútbol en relación a su posición en el campo de juego. Retos, 32, 58-61.

Fernández, J., Kazarez, M., Agazzi, B., \& Albín, S. (2014). Evaluación antropométrica según posición de juego de jugadores profesionales de fútbol uruguayo. Enfermería: Cuidados Humanizados, 3(2), 29-33. https://doi.org/ 10.22235/ech.v3i2.566

García, N., Zapata, D., Sáez, C., Yáñez, R., \& Peñailillo, L. (2015). Valoración de la masa grasa en fútbolistas jóvenes de alto rendimiento: comparación de métodos antropométricos con absorciometría dual de rayos $\mathrm{X}$ (DEXA). Arch. Med. Deporte, 32(168), 208-214.

García-Pinillos, F., Ruiz-Ariza, A., \& Latorre-Román, P. A. (2015). Influencia del puesto específico en la potencia y agilidad de jóvenes futbolistas. Retos. Nuevas tendencias en educación física, deporte y recreación, (27), 5861.

Gardasevic, J., Bjelica, D., \& Vasiljevic, I. (2019). Morphological Characteristics and Body Composition of Elite Soccer Players in Montenegro. International Journal of Morphology, 37(1), 284-288. http://dx.doi.org/10.4067/ S0717-95022019000100284

Gil-Gómez, J. \& Verdoy, P. J. (2011). Caracterización de deportistas universitarios de fútbol y baloncesto: antropometría y composición corporal. E-balonmano. com: Revista de Ciencias del Deporte, 7(1), 39-51.

Gómez, M., Ruiz, F., \& García, E. (2010). Actividades físicodeportivas que demandan los universitarios. Espiral. Cuadernos del Profesorado, 3(5), 3-10. Disponible en: http://www.cepcuevasolula.es/espiral.

Henríquez-Olguín, C., Báez, E., Ramírez-Campillo, R., \& Cañas, R. (2013). Perfil somatotípico del futbolista profesional chileno. International Journal of Morphology, 31(1), 225-230.

Hetzler, R. K., Schroeder, B. L., Wages, J. J., Stickley, C. D., \& Kimura, I. F. (2010). Anthropometry increases 1 repetition maximum predictive ability of NFL-225 test for Division IA college football players. The Journal of Strength, 24(6), 1429-1439. https:// doi.org/10.1519/JSC.0b013e3181d682fa

ISAK, Sociedad Internacional para el Avance de la Kineantropometría. (2001). Normas Internacionales para la Valoración Antropométrica. Potchefstroom, Sudáfrica. Librería Nacional de Australia.

Jorquera, C., Rodríguez, F., Torrealba, M.I., Campos, J., Gracia, N., \& Holway, F. (2013) Características Antropométricas de Futbolistas Profesionales Chilenos. International Journal of Morphology, 31(2), 609-614.

Leger, L.A. \& Lamber, J. I. (1982). A maximal multistage 20-m shuttle run test to predict VO2 max. European Journal of Applied Physiology and Occupational Physiology, 49(1), 1-12.

Manzi, V., Bovenzi, A., Impellizzeri, M. F., Carminati, I., \& Castagna, C. (2013). Individual training-load and aerobicfitness variables in premiership soccer players during the precompetitive season. The Journal of Strength \& Conditioning Research, 27(3), 631-636. https:// doi.org/ 10.1519/JSC.0b013e31825dbd81

Marfell, M., Olds, T., Stewart, A., \& Carter, L. (2006). International Standards for Anthropometric 
Assessment. Shenzhen, China, Shenzhen University Press.

Nicolozakes, C. P., Schneider, D. K., Roewer, B. D., Borchers, J. R., \& Hewett, T. E. (2018). Influence of Body Composition on Functional Movement Screen ${ }^{\mathrm{TM}}$ Scores in College Football Players. Journal of Sport Rehabilitation, 27(5), 431-437.

Nikolaidis, P. T. (2015). Can maximal aerobic running speed be predicted from submaximal cycle ergometry in soccer players? The effects of age, anthropometry and positional roles. Advanced Biomedical Research, 4(22). https:// doi.org/10.410372277-9175.166649

Peña-Reyes, M. E. (2017). El estudio del deporte en antropología física. Del somatotipo al fenómeno social. Cuicuilco Revista de Ciencias Antropológicas, 68, 59-76. Disponible en: http://www.scielo.org.mx/pdf/crca/v24n68/24488488-crca-24-68-59.pdf

Poveda, J. \& Casamichana, D. (2017). Rendimiento físico del fútbolista en entrenamientos y competición. Revista de Preparación Física en el Fútbol, 25(3), 10-22.

Rodríguez, A. N., Montenegro, O., \& Petro, J. L. (2019). Perfil dermatoglífico y somatotipificación de jugadores adolescentes de fútbol (Dermatoglyphic profile and somatotyping of adolescent soccer players). Retos, 36(36), 32-36.

Ryan-Stewart, H., Faulkner, J., \& Jobson, S. (2018). The influence of somatotype on anaerobic performance. PloS one, 13(5).

Sánchez-Ureña, B., Ureña-Bonilla, P., Salas-Cabrera, J., Blanco-Romero, L., \& Araya-Ramírez, F. (2011). Perfil Antropométrico y Fisiológico en Futbolistas de Élite Costarricenses según Posición de Juego. Revista Grupo sobreentrenamiento PubliCE Standard. Disponible en https://g-se.com/perfil-antropometrico-y-fisiologico-en- futbolistas-de-lite-costarricenses-segun-posicion-dejuego-1382-sa-B57cfb27205da8

Sargent, D. A. (1921). Physical test of man. American Physical Education Review, 26(4), 188-194, doi: 10.1080/ 23267224.1921.10650486

Stewart,A., Marfell-Jones, M., Olds, T., \& De Ridder, H. (2011). International standards for anthropometric assessment. New Zealand: ISAK, Lower Hutt.

Tomkinson, G. R., Popoviæ, N., \& Martin, M. (2003). Bilateral symmetry and the competitive standard attained in elite and sub-elite sport. Journal of Sports Sciences, 21(3), 201-211. https:// doi.org/10.1080/0264041031000071029a.

Vescovi, J. D. \& McGuigan, M. R. (2008). Relationship between sprinting, agility, and jump ability in female athletes. Journal of Sports Sciences, 26(1), 97-107.

Vidarte Claros, J. A., Castiblanco, H. D., Villa Barco, J. W., \& Ortega Parra, A. J. (2019). Values of the resistance of the university football player in special conditions, of the city of Manizales (Colombia) (Valores de la resistencia del jugador de fútbol universitario en condiciones especiales, de la ciudad de Manizales (Colombia)). Retos. Nuevas tendencias en Educación Física, Deportes y Recreación, 36(36), 211-215.

Wilmore, J. H. \& Behnke, A. R. (1969). An anthropometric estimation of body density and lean body weight in young men. Journal of Applied Physiology, 27(1), 25-31. https:/ /doi.org/10.1152/jappl.1969.27.1.25.

Zuñiga, G. U., Osorio, G. A., Toledo, D. I. D. J., \& Herrera, P. R. (2018). Somatotipo enfutbolistas mexicanos profesionales de diferente nivel competitivo. Retos. Nuevas tendencias en Educación Física, Deporte y Recreación, (34),100-102.

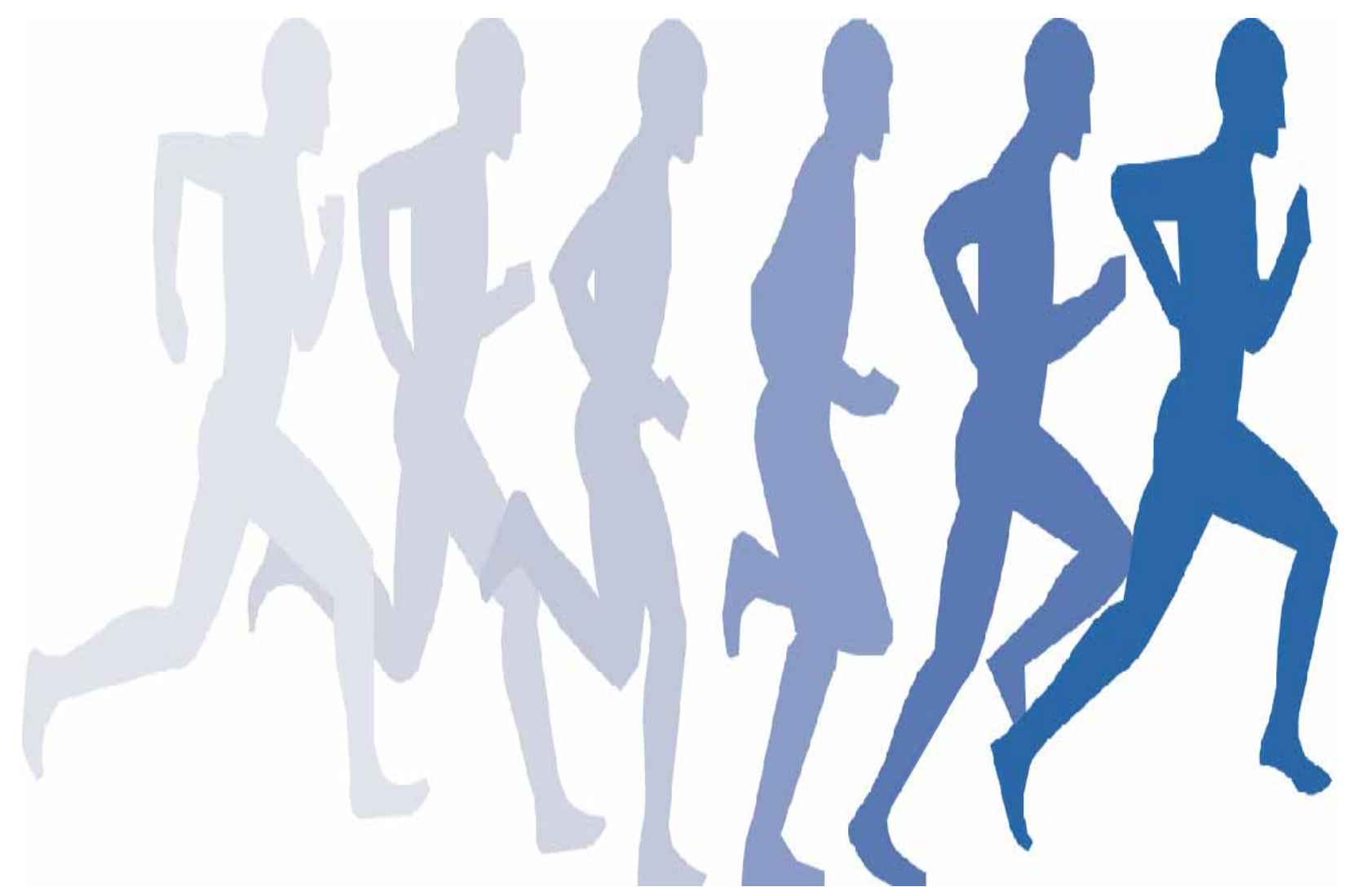

